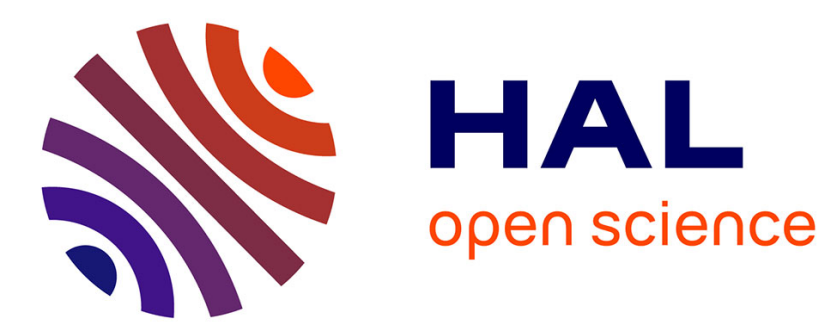

\title{
Strong coupling between phonons and optical beating in backward Brillouin scattering
}

\author{
Kien Phan Huy, Jean-Charles Beugnot, Joël-Cabrel Tchahame, Thibaut \\ Sylvestre
}

\section{- To cite this version:}

Kien Phan Huy, Jean-Charles Beugnot, Joël-Cabrel Tchahame, Thibaut Sylvestre. Strong coupling between phonons and optical beating in backward Brillouin scattering. Physical Review A: Atomic, molecular, and optical physics [1990-2015], 2016, 94, pp.43847 - 43847. 10.1103/PhysRevA.94.043847 . hal-01469831

\section{HAL Id: hal-01469831 \\ https://hal.science/hal-01469831}

Submitted on 16 Feb 2017

HAL is a multi-disciplinary open access archive for the deposit and dissemination of scientific research documents, whether they are published or not. The documents may come from teaching and research institutions in France or abroad, or from public or private research centers.
L'archive ouverte pluridisciplinaire HAL, est destinée au dépôt et à la diffusion de documents scientifiques de niveau recherche, publiés ou non, émanant des établissements d'enseignement et de recherche français ou étrangers, des laboratoires publics ou privés. 


\title{
Strong coupling between phonons and optical beating in backward Brillouin scattering
}

\author{
Kien Phan Huy, ${ }^{*}$ Jean-Charles Beugnot, Joël-Cabrel Tchahame, and Thibaut Sylvestre \\ Institut FEMTO-ST UMR 6174, Université Bourgogne Franche-Comté, CNRS, Besançon, France
}

(Received 12 July 2016; published 25 October 2016)

\begin{abstract}
Brillouin scattering is a fundamental nonlinear interaction between two optical waves and an acoustic wave mediated by electrostriction and photoelasticity. In this paper, we revisit the usual theory of this inelastic scattering to get a joint system in which the acoustic wave is strongly coupled to the interference pattern between the optical waves. We show in particular that when the coupling rate exceeds the phonon damping rate, the system enters the strong-coupling regime, giving rise to anticrossing in the dispersion relation and Rabi-like splitting. We further find numerically that strong coupling can, in principle, be observed using backward Brillouin scattering in subwavelength-diameter optical waveguides.
\end{abstract}

DOI: 10.1103/PhysRevA.94.043847

\section{INTRODUCTION}

Cavity optomechanics, a branch of physics that studies the interaction of light with tiny mechanical objects, has recently drawn widespread interest because of key fundamental observations such as resolved-sideband cooling, optomechanically induced transparency, quantum coherent coupling, and Rabi oscillations [1-4]. Rabi oscillations were initially described as damped periodic oscillations of an excited atom coupled to an electromagnetic cavity in which the atom alternately emits and reabsorbs photons [5]. From a theoretical point of view, this remarkable and ubiquitous phenomenon can be readily described as a joint system of two coupled oscillators. If both oscillators are uncoupled, they share the same degenerate eigenfrequency. When they are strongly coupled, however, the degeneracy is removed and the frequency is split into two distinguishable eigenfrequencies corresponding to the odd and even supermodes of the joint system. The frequency difference between the two eigenfrequencies is called the Rabi frequency $[5,6]$. When the same effect occurs at the quantum level, e.g., an atom and a photon embedded in a cavity, the new eigensolutions are coherent superpositions of the two particles. When the two particles are not interacting with each other, they have their own dispersion relations $k_{i}(\omega)$ that may cross each other. However, when the strong-coupling regime is reached, the joint system dispersion relation displays an avoided crossing and Rabi splitting. Since its first observation, this concept has recently been extended to cavity optomechanics where mechanical and optical modes of high-quality-factor resonators are strongly coupled [7,8], and to many other joint systems including photons, phonons, excitons, and plasmons [2,5,6,9-11].

In this work, we theoretically investigate strong coupling in backward Brillouin scattering (BS), which is a well-known photon-phonon interaction involving two frequency-detuned counterpropagating optical waves and an acoustic wave. Although similar ideas have recently been introduced by Van Laer et al. [12], our work specifically demonstrates that strong coupling can be achieved between the acoustic phonon and the optical beat note formed by the pump and Stokes waves. The strong coupling investigated in this paper fundamentally

\footnotetext{
*kphanhuy@univ-fcomte.fr
}

differs from other regimes because it is cavityless and does not rely on the optical and acoustic field variations. In our study, this is the phase difference between the pump and Stokes waves that govern the strong coupling. When the proper phase conditions are fulfilled, strong coupling and Rabi splitting can be numerically observed in subwavelength waveguides providing that the coupling rate exceeds the phonon damping rate.

The paper is organized as follows: First, we describe the principle and methodology of the photon-phonon interaction under consideration. Then we derive from the standard theory of BS a joint two-level system, as commonly used in the Rabi problem. Finally, we investigate from this system the specific conditions that allow the strong optoacoustic coupling regime to be achieved in small optical waveguides.

\section{PRINCIPLE AND METHODOLOGY}

Let us first describe the photon-phonon interaction under consideration. When a coherent laser light is coupled and guided into a thin optical fiber, as shown schematically in Fig. 1, light generates and interacts with several types of acoustic waves [13-15]. Here we assume only one acoustic longitudinal wave interacting with the guided light (this assumption will be discussed later on). As sketched in Fig. 1, $\mathrm{BS}$ is an inelastic scattering whereby two frequency-detuned optical pump (red right-pointing arrow) and Stokes (blue left-pointing arrow) waves coherently interact in a dielectric waveguide, giving rise to an optical interference pattern (purple) that generates an acoustic wave (green phonon arrow) through the effect of electrostriction. Simultaneously, the photoelastic effect creates a moving index grating that travels at the speed of hypersound (i.e., a few thousand of $\mathrm{m} \mathrm{s}^{-1}$ in silica and chalcogenide glasses). The index grating acts as a moving Bragg mirror and reflects off the pump light with a Doppler downshift matching the frequency detuning between the two optical waves. When the frequency detuning is equal to the acoustic phonon frequency, phase matching is achieved and therefore one gets amplification of both the Stokes wave and the optical beat note. In the weak-coupling regime, both the optical beat note and acoustic wave grow along the propagation distance, while in the strong-coupling regime, they couple and alternately exchange energy over short periods of few tens of microns. 


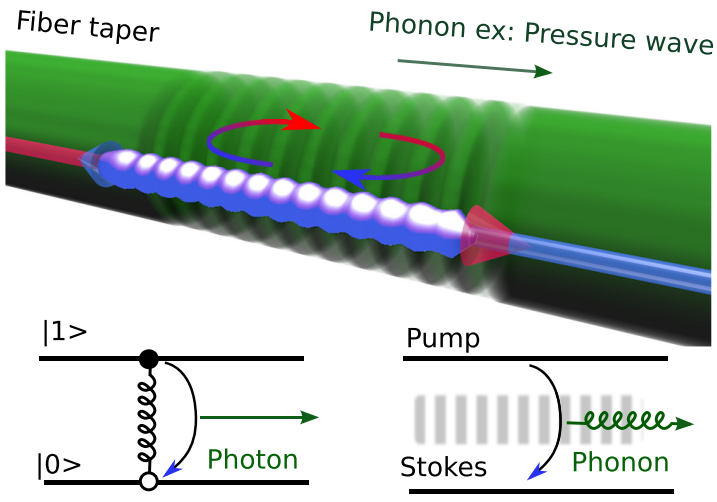

FIG. 1. Principle of strong coupling using backward Brillouin scattering in an optical fiber. A strong pump wave (red arrow, pointing right) coherently interacts with a counterpropagating probe wave, giving rise to an optical beat note which generates an acoustic wave through electrostriction. Under strong coupling, the interference pattern and the phonon are coherently coupled and alternately exchange energy over short periods of few tens of microns. Bottom left: scheme of a two-level system with an exciton interacting with a photon. Bottom right: scheme of the strong-coupling regime in Brillouin scattering.

From a quantum point of view, a pump photon is annihilated to create both a Stokes photon and an acoustic phonon, as depicted in the bottom left of Fig. 1. Similarly to the Rabi problem, the two pump and Stokes photons can be seen as the two levels of an artificial atom. In our case, however, the two coupled objects are actually the acoustic phonon and the optical beat between the pump and Stokes waves. As a result, our joint system is fundamentally different from the conventional exciton-polariton picture. In the latter case, the two-level system includes only one particle. It is described as an exciton with a given resonant frequency, as sketched by the spring in the bottom left of Fig. 1. The photon interacting with the exciton is actually a signal probe tuned at the resonant frequency. The experiment usually consists of tuning the probe signal by adjusting its angle (wave vector) or changing the temperature to affect the resonant frequency of the exciton. In the BS case, the pump and Stokes light replace the two levels since $\omega_{P}>\omega_{S}$ (see bottom right of Fig. 1). However, the levels are not bounded by a resonant frequency, as defined by the gap of a two-level atom. In BS, the Brillouin frequency shift is given by the phase-matching condition between the acoustic properties of the mechanical structure formed by the optical fiber and the optical waves. The resonant nature of the process mostly depends on the acoustic properties of the waveguide. This is illustrated in the bottom right of Fig. 1, where the acoustic phonon plays the role of the spring. Since the pump and Stokes waves are coherent laser light that are conveniently produced and manipulated, we suggest to use them for probing the coupling with the acoustic phonon.

To observe Rabi-like oscillations in such a two-level system, it is also required to limit the interaction with, ideally, one guided acoustic mode, which is not possible using bulky standard optical fibers. This mode selection can, however, be achieved using an optical fiber tapered down to a subwavelength diameter [14] or a silicon nanoscale waveguide [16]. To this end, we solved the dispersion equation of longitudinal acoustic modes $\beta_{a}(\Omega)$ in a silica fiber taper in order to select only one acoustic mode interacting with guided light. This dispersion relation reads [15]

$$
\begin{aligned}
& \frac{2 p}{a}\left(q^{2}+\beta_{a}^{2}\right) J_{1}(p a) J_{1}(q a)-\left(q^{2}-\beta_{a}^{2}\right)^{2} J_{0}(p a) J_{1}(q a) \\
& \quad=4 \beta_{a}^{2} p q J_{1}(p a) J_{0}(q a)
\end{aligned}
$$

with $p=\sqrt{\frac{\Omega^{2}}{V_{L}^{2}}-\beta_{a}^{2}}$ and $q=\sqrt{\frac{\Omega^{2}}{V_{T}^{2}}-\beta_{a}^{2}}$, where $V_{L}$ and $V_{T}$ are the longitudinal and shear acoustic velocities, respectively, related to the longitudinal and shear components of the displacement. $J_{i}$ are the Bessel functions. $a$ is the fiber taper radius. We then do the same work for the optical modes, as in Ref. [17]. The optical dispersion equation for a step index fiber of core refractive index $n_{1}$ and cladding index $n_{2}$ is

$$
\begin{aligned}
& \left\{\frac{J_{m}^{\prime}\left(\gamma_{1} a\right)}{\gamma_{1} a J_{m}\left(\gamma_{1} a\right)}+\frac{n_{1}^{2}}{n_{2}^{2}} \frac{K_{m}^{\prime}\left(\gamma_{2} a\right)}{\gamma_{2} a K_{m}\left(\gamma_{2} a\right)}\right\} \\
& =\frac{m}{a^{2}}\left(\frac{1}{\gamma_{1}^{2}}+\frac{1}{\gamma_{2}^{2}}\right)\left(\frac{1}{\gamma_{1}^{2}}+\frac{n_{2}^{2}}{n_{1}^{2}} \frac{1}{\gamma_{2}^{2}}\right),
\end{aligned}
$$

where $\gamma_{1}=\sqrt{k_{0}^{2} n_{1}^{2}-\beta^{2}}, \gamma_{2}=\sqrt{\beta^{2}-k_{0}^{2} n_{2}^{2}}$, and $K_{m}$ denotes the modified Bessel function of the second kind, with the prime denoting differentiation with respect to the argument. $k_{0}$ is the propagating wave vector in vacuum. For each integer value $m$, the eigenvalue $\beta$ is the effective propagation constant along the fiber axis of the given mode. This leads to the propagating constants of the pump $(P)$ and Stokes $(S)$ waves, $\beta_{P}(\omega)$ and $\beta_{S}(\omega)$. Then the phase-matching condition $\beta_{P}(\omega+\Delta \Omega)-\beta_{S}(\omega)=\beta_{a}(\Delta \Omega)$ sets the detuning frequency $\Delta \Omega$ at which Brillouin scattering occurs.

Figure 2 typically shows a numerical simulation of the acoustic wave spectrum generated in a silica fiber taper (see Appendix A for chalcogenide), as a function of frequency detuning $\Delta \Omega$ and of the fiber diameter $[14,15]$. The color plot actually shows the overlap between acoustic and optical modes and it corresponds to the Brillouin scattering efficiency. As can be seen, there are several acoustic waves in the frequency range 5-13 GHz [14]. We can also see several anticrossings in Fig. 2 that appear when a pressure branch (dotted line) crosses a shear branch (dashed line). This is due to the fact that longitudinal modes in acoustic waveguides exhibit both pressure and shear components coupled at waveguide boundaries. This coupling leads to the anticrossing seen in Fig. 2 and is fundamentally different from the strong coupling described in this paper. We note also that around $5 \mathrm{GHz}$, there is no avoided crossing between the two surface Rayleigh waves because they have orthogonal polarizations. The squared red area in Fig. 2 shows that for a submicron-diameter fiber, there is a small frequency range where we can isolate a single acoustic mode around $8 \mathrm{GHz}$. In our study, we investigate the strong-coupling regime in a region such as this red area.

In addition, it is also important to have a sufficiently strong coupling strength for transferring the energy back and forth between the optical beat note and the phonon. As a result, large Brillouin efficiency is also required, which is the case in a subwavelength waveguide due to the strong light confinement. Moreover, recent works have shown that Brillouin gain 


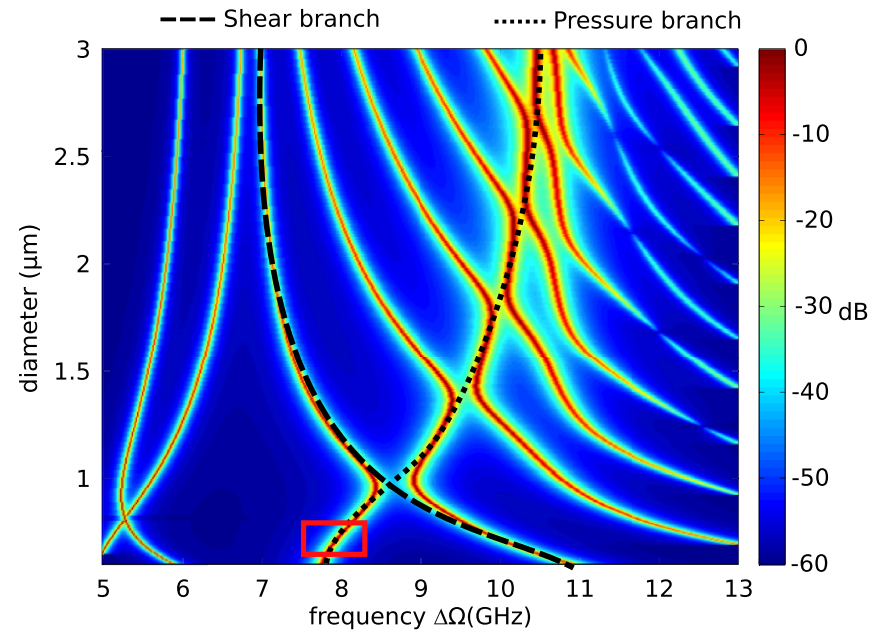

FIG. 2. Color plot of the Brillouin spectrum related to the coupling strength between longitudinal acoustic modes with the fundamental optical mode of a silica fiber taper as a function of the acoustic frequency (horizontal axis) and of the taper diameter (vertical axis). The color scale describes the normalized overlap between the acoustic and optical modes. The scale is referenced with $0 \mathrm{~dB}$ corresponding to the usual Brillouin gain in silica fibers, $\gamma=3.3 \times 10^{-11} \mathrm{~m} \mathrm{~W}^{-1}$. The absolute effective area $A_{\text {eff }}$ is not taken into account; it would show an enhancement for small diameters. Several avoided crossings appear when a pressure branch (dotted line) crosses a shear branch (dashed line). The squared red area isolates a single acoustic mode, required for the strong-coupling regime.

could be significantly enhanced in nanoscale and large step index waveguides when radiation pressure enters into play or becomes comparable to the electrostrictive force $[18,19]$.

\section{THEORY}

In the following, we rederive the usual theory of BS [20] to yield a joint system that can experience Rabi-like oscillations. The pump $(P)$ and Stokes $(S)$ fields and the material density $\tilde{\rho}$ can be expressed as

$$
\begin{gathered}
\tilde{E_{P}}(x, y, z, t)=\tilde{A}_{P}(z, t) E_{P}(x, y) e^{i\left(\beta_{P} z-\omega_{P} t+\varphi_{P}\right)}, \\
\tilde{E}_{S}(x, y, z, t)=\tilde{A}_{S}(z, t) E_{S}(x, y) e^{i\left(\beta_{S} z-\omega_{S} t+\varphi_{S}\right)}, \\
\tilde{\rho}(x, y, z, t)=\rho_{0}+\tilde{\rho}(z, t) \mathcal{R}(x, y) e^{i\left(\beta_{a} z-\Omega_{a} t+\varphi_{a}\right)},
\end{gathered}
$$

where $\rho_{0}$ is the mean density of the medium, $\beta_{P, S, a}, \omega_{P, S}$, and $\Omega_{a}$, and $\varphi_{P, S, a}$ are the wave vectors, frequencies, and phases of the optical and acoustic waves, respectively. $\tilde{A}_{P, S}$, $\tilde{\rho}(z, t)$ are slowly varying envelopes, and $E_{P, S}$ and $\mathcal{R}$ are the transverse profiles of each wave. The backward propagation of the Stokes wave is taken into account with $\beta_{S}<0$ and the complex conjugates are omitted. We note that this model of a single-plane acoustic wave readily assumes that only one acoustic wave can be possibly coupled to the optical waves, as in Fig. 2. Moreover, effects due to strong light confinement do not explicitly appear but can be taken into account, as discussed in Appendix B. We also neglect the anti-Stokes scattering and the second-order Stokes scattering [20]. That assumption is also discussed in Appendix F. By including the above equations in the wave equation, one gets [20]

$$
\begin{gathered}
\frac{\partial \tilde{A}_{P}}{\partial z}+\frac{\partial \beta}{\partial \omega} \frac{\partial \tilde{A}_{P}}{\partial t}=\frac{i \omega_{P} \gamma_{e}}{2 n_{P} c \rho_{0}} \frac{1}{D_{\mathrm{eff}}} \tilde{\rho} \tilde{A}_{S}, \\
-\frac{\partial \tilde{A}_{S}}{\partial z}+\frac{\partial \beta}{\partial \omega} \frac{\partial \tilde{A}_{S}}{\partial t}=\frac{i \omega_{S} \gamma_{e}}{2 n_{S} c \rho_{0}} \frac{1}{D_{\mathrm{eff}}} \tilde{\rho}^{*} \tilde{A}_{P},
\end{gathered}
$$

where $\gamma_{e}$ is the electrostriction constant, $n_{P}, n_{S}$ are the refractive indices for the pump and Stokes waves, respectively, $c$ is the speed of light in vacuum, and $D_{\text {eff }}$ is a factor coming from normalization (for details, see Appendix B). We note that the above coupled-amplitude equations can experience strong coupling provided that $|\tilde{\rho}|$ is constant, as discussed in [12]. In this case, temporal oscillations of $\tilde{A}_{P}$ and $\tilde{A}_{S}$ can occur in a cavity configuration where stationary waves are present. Our aim here is to show a different strong-coupling regime between the acoustic phonon and the optical interference pattern. For that purpose, we will hereafter combine Eqs. (6) and (7) to get the evolution of the optical beat note.

\section{A. Poynting vector: Optical beat note}

Since the optical beat note is an intensity modulation induced by the coherent superposition of pump and Stokes waves, it can be described by the $z$ component of the Poynting vector as

$$
\begin{aligned}
\vec{S} & =\vec{E} \times \vec{H}, \\
& =\vec{S}_{P}+\vec{S}_{S}+\vec{E}_{P} \times \vec{H}_{S}+\vec{E}_{S} \times \vec{H}_{P},
\end{aligned}
$$

where $\vec{S}_{P, S}$ are the Poynting vectors for the pump and Stokes waves. The two first terms of the right-hand side (RHS) of Eq. (9) describe the independent Stokes and pump wave Poynting vectors. They are linked to power evolution by the flux $\Phi=\int_{S} \vec{S} d \vec{s}$. The two last terms in the RHS display the coherent local intensity modulation due to interference. Those terms do not describe a power flow since they vanish after integration; however, they describe a local intensity fluctuation, i.e., the optical beat note. Using the expressions of the pump and Stokes waves, given by Eqs. (3) and (4), we can readily see that this intensity fluctuations varies as $e^{i(\Delta \beta z-\Delta \omega t)}$, where $\Delta \beta=\beta_{P}-\beta_{S}$ and $\Delta \omega=\omega_{P}-\omega_{S}$. The optical beat note thus travels at a speed defined by $v_{b}=\frac{\Delta \omega}{\Delta \beta}$. It is around a few thousands $\mathrm{ms}^{-1}$, the same speed as the acoustic wave when phase matching is satisfied. In the following, we will transform Eqs. (6) and (7) to derive an equation of the optical beat note and its relationship with the phonon. Careful attention will be paid to the omitted complex conjugate terms; however, all the terms of Eq. (9) are not detailed and the focus is put only on phase-matched terms. First, we introduce the amplitude of the magnetic field $\tilde{B}_{P}$. In the plane-wave approximation, it gives, for the pump wave, $\tilde{B}_{P}(z, t)=\frac{\beta_{P}}{\omega_{P} \mu_{0}} \tilde{A}_{P}(z, t)$. Substituting it into Eqs. (6) and (7) yields

$$
\begin{gathered}
\frac{\partial \tilde{B}_{P}}{\partial z}+\frac{\partial \beta}{\partial \omega} \frac{\partial \tilde{B}_{P}}{\partial t}=\frac{\beta_{P}}{\beta_{S}} \frac{i \omega_{P} \gamma_{e}}{2 n_{P} c \rho_{0}} \frac{1}{D_{\mathrm{eff}}} \tilde{\rho} \tilde{B}_{S}, \\
\frac{\partial \tilde{B}_{S}}{\partial z}-\frac{\partial \beta}{\partial \omega} \frac{\partial \tilde{B}_{S}}{\partial t}=-\frac{\beta_{S}}{\beta_{P}} \frac{i \omega_{S} \gamma_{e}}{2 n_{S} c \rho_{0}} \frac{1}{D_{\mathrm{eff}}} \tilde{\rho}^{*} \tilde{B}_{P} .
\end{gathered}
$$


Multiplying Eq. (6) by $\tilde{B}_{S}^{*}$ and the conjugate of Eq. (11) by $\tilde{A}_{P}$, we find

$$
\begin{aligned}
& \frac{\partial \tilde{A}_{P}}{\partial z} \tilde{B}_{S}^{*}+\frac{\partial \beta}{\partial \omega} \frac{\partial \tilde{A}_{P}}{\partial t} \tilde{B}_{S}^{*}=\frac{i \omega_{P} \gamma_{e}}{2 n_{P} c \rho_{0}} \frac{1}{D_{\mathrm{eff}}} \tilde{\rho} \tilde{A}_{S} \tilde{B}_{S}^{*}, \\
& \tilde{A}_{P} \frac{\partial \tilde{B}_{S}^{*}}{\partial z}-\frac{\partial \beta}{\partial \omega} \tilde{A}_{P} \frac{\partial \tilde{B}_{S}^{*}}{\partial t}=\frac{\beta_{S}}{\beta_{P}} \frac{i \omega_{S} \gamma_{e}}{2 n_{S} c \rho_{0}} \frac{1}{D_{\mathrm{eff}}} \tilde{\rho} \tilde{A}_{P} \tilde{B}_{P}^{*} .
\end{aligned}
$$

Since $P$ and $S$ waves are counterpropagating and shifted in frequency by only a few $\mathrm{GHz}$, we can make the following assumptions: $\frac{\beta_{S}}{\beta_{P}} \simeq-1$ and $n=n_{S} \simeq n_{P}$. Taking the sum of the above equations, and neglecting the group velocity, as explained in Appendix C, we readily find

$$
\frac{\partial \tilde{A}_{P} \tilde{B}_{S}^{*}}{\partial z}=-\frac{i \omega_{P} \omega_{S} \gamma_{e} \tilde{\rho}}{n c \rho_{0} D_{\mathrm{eff}}}\left(\frac{\tilde{A}_{P} \tilde{B}_{P}^{*}}{\omega_{P}}-\frac{\tilde{A}_{S} \tilde{B}_{S}^{*}}{\omega_{S}}\right) .
$$

On the RHS of Eq. (12), we see that the optical beat note is clearly coupled with the phonon $\tilde{\rho}$. The term within brackets is usually referred to as the "population inversion" in a two-level system problem. Indeed, if both waves were propagating in the same direction, each $\frac{\tilde{A}_{k} \tilde{B}_{k}^{*}}{\omega_{k}}$ term would be proportional to the power $P_{k}$, and be denoted as the "population" of level $k$. However, in the specific case of counterpropagating waves, the name population inversion is not appropriate. Since each population term is proportional to its Poynting vector, its sign depends on the direction of the power flow along the $z$ axis. If the Stokes wave propagates in the backward direction (BS), the flux of the Poynting vector for the Stokes wave is negative, leading to $\frac{2 \tilde{A}_{S} \tilde{B}_{S}^{*}}{\omega_{S}}=-\frac{P_{S}}{\omega_{S}}$. As a result, the term in brackets of the RHS of Eq. (12) should be named "total population." This situation is very specific to the present work, and does not occur in copropagating or static cases such as the atom-photon coupling. In the atom-photon picture, the conservation law stipulates that the sum of the populations is constant, $\dot{\rho}_{11}+\dot{\rho}_{22}=0$ [20]. However, for counterpropagating waves (pump and Stokes), the Poynting picture shows that the conservation through a transverse plane turns to $\dot{\Phi}_{P}-\dot{\Phi}_{S}=0$, which is unusual when compared to the atom-photon picture (see Appendix D).

In order to build a joint system where the optical beat is coupled with the phonon, it is important that the coupling rate does not vary or, in other words, that the total population is nearly constant. To this end, we assume that anti-Stokes and second-order Stokes scattering are negligible (see Appendix F). Under this assumption, we can multiply Eq. (12) with the previously omitted fast oscillating phase term $e^{i(\Delta \beta z-\Delta \omega t)}$, add and subtract $-i \Delta \beta \tilde{E}_{P} \tilde{H}_{S}^{*}(z, t)$, simplify with Eq. (3), and take the Fourier transform, to get

$$
\frac{\partial \widehat{\tilde{E}_{P} \tilde{H}_{S}^{*}}(z, \Omega)}{\partial z}-i \Delta \beta \widehat{\tilde{E}_{P} \tilde{H}_{S}^{*}}(z, \Omega)=\frac{k_{o p}}{2} \hat{\rho}(z, \Omega),
$$

with $\tilde{E}_{P} \tilde{H}_{S}^{*}(z, t)=\tilde{A}_{P} \tilde{B}_{S}^{*}(z, t) e^{i(\Delta \beta z-\Delta \omega t)}$ and the hat denotes the Fourier transform. The coupling coefficient is $k_{o p}=$ $-\frac{\omega_{P} \omega_{S} \gamma_{e}}{2 n c \rho_{0} D_{\text {eff }}}\left\{\frac{P_{P}}{\omega_{P}}+\frac{P_{S}}{\omega_{S}}\right\}$. This equation actually describes the optical beat note in the Fourier domain, with $\Omega$ the frequency detuning between the pump and Stokes waves. If the RHS is neglected, meaning that there is no nonlinear interaction, this equation becomes the dispersion equation of the optical beating. We can then see that the optical beating travels with the wave vector $\Delta \beta$ that is related to the speed $v=\frac{\Delta \omega}{\Delta \beta}$.

\section{B. Acoustic equation}

To build the other half of the joint system, a similar equation must be derived for the acoustic phonon. Using a standard description of a pressure wave for the acoustic model, we get

$$
\begin{aligned}
& -2 i \Omega \frac{\partial \tilde{\rho}}{\partial t}+\left(\Omega_{a}^{2}-\Omega^{2}-i \Omega \Gamma_{B}\right) \tilde{\rho}-2 i \beta_{a} v_{a}^{2} \frac{\partial \tilde{\rho}}{\partial z} \\
& =-\frac{\gamma_{e} \beta_{a}^{2}}{n c} \frac{1}{D_{\mathrm{eff}}} \tilde{A}_{P} \tilde{B}_{S}^{*} .
\end{aligned}
$$

The first and third terms of the left-hand side (LHS) of Eq. (14) show that the acoustic wave travels at the speed $v_{a}=\frac{\Omega_{a}}{\beta_{a}}$. The second term of the LHS is the mechanical stresses induced by the phonon. At the resonant frequency $\Omega_{a}$, only the $\Gamma_{B}$ term related to the damping is left. The RHS of Eq. (14) provides the coupling rate with the optical beat note. To write this equation, we assume that the phase matching for backward BS is satisfied and therefore that the acoustic wave vector is twice the optical one, as $\beta_{a}=2 \beta_{P}$. Now, we should describe the whole dispersion curve and not restrict our study to the phasematching case only. We will thus not make this assumption and let $\beta_{a}(\Omega)$ vary in the surrounding of frequency resonance $\left(\Omega-\Omega_{a} \ll \Omega_{a}\right)$. The $\Omega_{a}^{2}-\Omega^{2}-i \Omega \Gamma_{B}$ term then reduces to $-i \Omega \Gamma_{B}$, which stands for the acoustic phonon losses.

To get the dispersion relation of the phonon, we rewrite the above equation by keeping the fast oscillating phase term $e^{i\left(\beta_{a} z-\Omega_{a} t\right)}$. We then add and subtract $i \beta_{a} \tilde{\rho} e^{i\left(\beta_{a} z-\Omega_{a} t\right)}$ and straightforwardly get

$$
\begin{aligned}
& \left\{-i \beta_{a} \tilde{\rho}+\frac{\Omega}{\Omega_{a}} \frac{1}{v_{a}} \frac{\partial \tilde{\rho}}{\partial t}\right\} e^{i\left(\beta_{a} z-\Omega_{a} t\right)}+\frac{\partial \tilde{\rho}_{\phi}}{\partial z} \\
& \simeq i u(\Omega) \tilde{\rho}_{\phi}+i k_{p o} \tilde{E}_{P} \tilde{H}_{S}^{*}
\end{aligned}
$$

where $\tilde{\rho}_{\phi}=\tilde{\rho}(z, t) e^{i\left(\beta_{a} z-\Omega_{a} t\right)}+$ c.c. and

$$
\begin{gathered}
u(\Omega)=\frac{i \Omega \Gamma_{B}}{2 \beta_{a} v_{a}^{2}} \\
k_{p o}=-\frac{1}{2 \beta_{a} v_{a}^{2}} \frac{\gamma_{e} \beta_{a}^{2}}{n_{S} c} \frac{1}{D_{\mathrm{eff}}}
\end{gathered}
$$

are the acoustic losses and the nonlinear coupling rate, respectively. The dispersion relation can be derived from the Fourier transform of Eq. (15). Assuming that the detuning from the Brillouin frequency shift is low, $\Omega-\Omega_{a} \ll \Omega_{a}$, we find

$$
\begin{aligned}
& \frac{\partial \hat{\rho}(z, \Omega)}{\partial z}-i \beta_{a}(\Omega) \hat{\rho}(z, \Omega) \\
& \quad=i u(\Omega) \hat{\rho}(z, \Omega)+i k_{p o} \widehat{\tilde{E}_{P} \tilde{H}_{S}^{*}}(z, \Omega),
\end{aligned}
$$

where $\beta_{a}(\Omega)=\beta_{a}+(\Omega-\Delta \omega) \frac{1}{v_{a}}$ is the dispersion relation of the phonon and $\rho(z, \Omega)$ is the Fourier transform of $\tilde{\rho}_{\phi}$. 


\section{The joint system}

We now combine Eqs. (13) and (19) as a joint system with $2 \widetilde{\tilde{E}_{P} \tilde{H}_{S}^{*}}$ denoting the optical beat,

$$
\frac{\partial}{\partial z}\left(\begin{array}{c}
2 \widehat{\tilde{E}_{P} \tilde{H}_{S}^{*}} \\
\hat{\rho}
\end{array}\right)=i\left(\begin{array}{ll}
k_{o o} & k_{o p} \\
k_{p o} & k_{p p}
\end{array}\right)\left(\begin{array}{c}
2 \widehat{\tilde{E}_{P} \tilde{H}_{S}^{*}} \\
\hat{\rho}
\end{array}\right),
$$

where $k_{o o}=\Delta \beta$ and $k_{p p}=\beta_{a}(\Omega)+u(\Omega)$ are the eigenvalues of the uncoupled system, corresponding to the wave vectors of the optical beat note and of the phonon, respectively. Note that in the expression of $k_{p p}$, we have $u\left(\Omega_{a}\right)=i \frac{\Gamma_{B}}{2 v_{a}}$ that is related to the Brillouin linewidth $\Gamma_{B}$, and thus the phonon decay rate or lifetime. Since this term is imaginary, it gives an imaginary part to $k_{p p}$ that stands for the phonon propagation losses. The coupling coefficient $k_{p o}$ was previously defined in Eq. (17). The eigenvalues $K_{ \pm}$of the joint system are solutions of the characteristic polynomial as

$$
\left(K_{ \pm}-k_{o o}\right)\left(K_{ \pm}-k_{p p}\right)-k_{o p} k_{p o}=0 .
$$

If $k_{o p} k_{p o}=0$, the coupling rate is zero and there are two independent eigenvalues with dispersion curves that cross each other. If the coupling term is nonzero, it is more convenient to provide the equation in the form

$$
K_{ \pm}^{2}-\left(k_{o o}+k_{p p}\right) K_{ \pm}+k_{p p} k_{o o}-k_{o p} k_{p o}=0
$$

with

$$
k_{o p} k_{p o}=\frac{\beta_{a} \Gamma_{B} c}{8 n v_{a}} \frac{g_{0}}{A_{\text {eff }}}\left\{\frac{P_{P}}{\omega_{P}}+\frac{P_{S}}{\omega_{S}}\right\},
$$

where $g_{0}$ is the Brillouin gain defined in Appendix D, and $A_{\text {eff }}=D_{\text {eff }}^{2}$ is the effective area of the mode-field diameter (MFD). This equation possesses two solutions if the discriminant is positive,

$$
\Delta=\left(k_{o o}+k_{p p}\right)^{2}-4\left(k_{p p} k_{o o}-k_{o p} k_{p o}\right) \geqslant 0 .
$$

At the crossing of two dispersion curves $\left(\Omega=\Omega_{a}\right)$, where the phase-matching condition is fulfilled, $\Delta \beta=\beta_{P}-\beta_{S}=$ $\beta_{a}$, neglecting the phonon losses leads to $k_{o o}=k_{p p}$. We can rewrite this equation as

$$
\Delta=\left(k_{o o}-k_{p p}\right)^{2}+4 k_{o p} k_{p o} \geqslant 0 .
$$

Here we see that at the crossing point of the uncoupled dispersion curves, where the real part of $k_{o o}$ equals the real part of $k_{p p}$, only the coupling term and the imaginary parts of $k_{o o}$ and $k_{p p}$ still remain in the equation. Therefore, if the sum of the remaining terms is positive, the eigenvalues are

$$
K_{ \pm}=\frac{1}{2}\left\{k_{o o}+k_{p p} \pm \sqrt{\Delta}\right\} .
$$

We first compute the result of this equation, neglecting the phonon losses $u(\Omega)=0$. This is illustrated in Fig. 3 that shows the wave vector $K_{ \pm}$as a function of frequency detuning $\Omega$ for a $1-\mu \mathrm{m}$-diameter chalcogenide tapered optical fiber, with $40 \mathrm{~W}$ and $1 \mathrm{~mW}$ pump and Stokes power (for parameters, see Appendix E). The Rabi splitting is clearly visible in Fig. 3 as an avoided crossing between the optical beat note and phonon branches. Specifically, the splitting is characterized by a clear gap between the two curves of $\sqrt{\Delta}$. As a comparison, the dashed gray lines show the intersecting dispersion curves without any coupling. Note that for the simulation shown

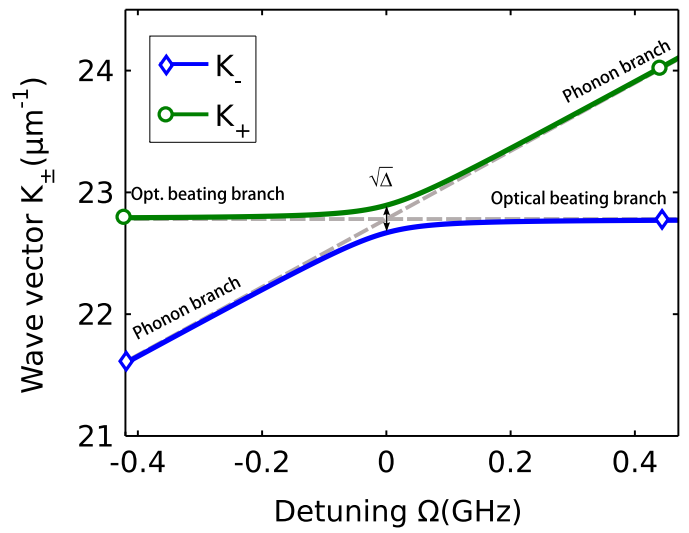

FIG. 3. Dispersion curves $K_{ \pm}(\Omega)$ showing acoustic Rabi splitting and anticrossing in a $1-\mu \mathrm{m}$-diameter $\mathrm{As}_{2} \mathrm{Se}_{3}$ tapered optical fiber with $40 \mathrm{~W}$ pump power and $1 \mathrm{~mW}$ Stokes power. The parameters are from Appendix E and the phonon losses were neglected. Dashed gray lines show the intersecting dispersion curves when there is no coupling.

here, we considered a chalcogenide glass fiber taper [13]. In the following, we will investigate the pump and Stokes equations to find what lies behind the constant total-population assumption we previously made.

\section{THE CONSTANT TOTAL-POPULATION ASSUMPTION}

Similar calculations can be done to describe the intensity fluctuations of both pump and Stokes waves,

$$
\begin{aligned}
& \frac{\partial \tilde{A}_{P} \tilde{B}_{P}^{*}}{\partial z}+\frac{\partial \beta}{\partial \omega} \frac{\partial \tilde{A}_{P} \tilde{B}_{P}^{*}}{\partial t}=\kappa\left\{\tilde{\rho} \tilde{A}_{S} \tilde{B}_{P}^{*}+\tilde{\rho}^{*} \tilde{A}_{P} \tilde{B}_{S}^{*}\right\}, \\
&-\frac{\partial \tilde{A}_{S} \tilde{B}_{S}^{*}}{\partial z}+\frac{\partial \beta}{\partial \omega} \frac{\partial \tilde{A}_{S} \tilde{B}_{S}^{*}}{\partial t}=\kappa\left\{\tilde{\rho}^{*} \tilde{A}_{P} \tilde{B}_{S}^{*}+\tilde{\rho} \tilde{A}_{S} \tilde{B}_{P}^{*}\right\},
\end{aligned}
$$

with $\kappa=\frac{i \omega \gamma_{e}}{2 n c \rho_{0}} \frac{1}{D_{\text {eff }}}$. Subtracting both equations, we can write in the steady-state regime the spatial evolution of the total population as

$$
\begin{aligned}
& \frac{\partial}{\partial z}\left(\frac{\tilde{A}_{P} \tilde{B}_{P}^{*}}{\omega_{P}}-\frac{\tilde{A}_{S} \tilde{B}_{S}^{*}}{\omega_{S}}\right) \\
& \quad=\frac{i \gamma_{e}}{2 n_{P} c \rho_{0}} \frac{1}{D_{\mathrm{eff}}} 2\left\{\tilde{\rho}^{*} \tilde{A}_{P} \tilde{B}_{S}^{*}+\tilde{\rho} \tilde{A}_{S} \tilde{B}_{P}^{*}\right\}=0 .
\end{aligned}
$$

The constant total-population assumption then implies that the RHS of the equation is zero, leading straightforwardly to

$$
\varphi_{a}=\varphi_{P}-\varphi_{S}+\pi[2 \pi],
$$

for nonzero fields. This is an important result because Stokes or anti-Stokes generation processes are generally associated with different phase relationships, as summarized in Table I. This table shows that in this phase configuration, neither the Stokes nor the anti-Stokes process is dominant, leading to nonfluctuating pump and Stokes power. This counterintuitive result is confirmed by Eqs. (26) and (27), where the RHS becomes zero. We must stress that it does not prevent the optical beat note from fluctuating. An optical beat is an interference fringe pattern that results from the coherent superposition of two waves, whether they are coupled or not. The Rabi splitting only describes the periodic loss of visibility of those fringes. 
TABLE I. Table of phase relationships in BS processes. Note that $\varphi_{P}-\varphi_{S}=\varphi_{a}[2 \pi]$ does not draw any interest since all amplitudes must be zero to prevent gain or depletion. We focus on $\varphi_{P}-\varphi_{S}=$ $\varphi_{a}+\pi[2 \pi]$ cases since they describe nonzero solutions.

\begin{tabular}{lcccc}
\hline \hline Process & Pump & Stokes & Phonon & Phase relationship \\
\hline Stokes & $\searrow$ & $\nearrow$ & $\nearrow$ & $\varphi_{P}-\varphi_{S}=\varphi_{a}-\frac{\pi}{2}[2 \pi]$ \\
Anti-Stokes & $\nearrow$ & $\searrow$ & $\searrow$ & $\varphi_{P}-\varphi_{S}=\varphi_{a}+\frac{\pi}{2}[2 \pi]$ \\
Rabi splitting & $\sim$ & $\sim$ & $\sim$ & $\varphi_{P}-\varphi_{S}=\varphi_{a}+\pi[2 \pi]$ \\
\hline \hline
\end{tabular}

The phenomenon is purely phase sensitive. If the phase is halfway between a constructive and destructive interference, thus the optical beat "disappears" without any change of the pump or the Stokes intensities. From an experimental point of view, it means that we need first to generate the phonon in a standard stimulated Brillouin scattering (SBS) configuration and then apply a phase shift using standard radio-frequency components to observe the Rabi splitting.

Now, we will take into account the phonon losses and see how it will affect the Rabi splitting. Furthermore, we will define a criterion for the strong-coupling regime and study how this criterion evolves with the material nonlinearity and the pump power.

\section{PHONON LOSSES AND SPLITTING RATIO}

In the last section, the imaginary parts of $k_{o o}$ and $k_{o p}$ were neglected, making each wave lossless. In the exciton-polariton picture, the weak- and strong-coupling regimes are usually separated by comparing the coupling rate $g$ and the excitonphoton decay rates $\gamma_{\mathrm{ph}}$ and $\gamma_{\mathrm{ex}}$. If $g \gg \gamma_{\mathrm{ex}}, \gamma_{\mathrm{ph}}$, the system is in the strong-coupling regime, whereas if $g \ll \gamma_{\mathrm{ex}}, \gamma_{\mathrm{ph}}$, the system is in the weak-coupling regime [21]. Our case using $\mathrm{BS}$ is very similar. If we do not neglect the phonon losses in (24), we get the following inequality:

$$
\sqrt{\Delta}=2 \sqrt{k_{o p} k_{p o}} \geqslant|u(\Omega)|,
$$

where the right-hand side $\sqrt{\Delta}$ plays a role similar to the coupling rate $g$, with the difference that our eigenvalues are wave vectors, and $\sqrt{\Delta}$ is thus homogeneous to $m^{-1}$, instead of $s^{-1}$. The LHS term $u(\Omega)$ defined by Eq. (16) is related to the phonon decay rate $\gamma_{\mathrm{ex}}$ in the exciton-polariton picture. To discriminate the weak-coupling from the strongcoupling regime, it is convenient to rewrite Eq. (30) with the splitting ratio $p=\frac{\sqrt{\Delta}}{|u(\Omega)|}$. If $p \ll 1$, the joint system is in the weak-coupling regime, whereas if $p \gg 1$, it is in the strong optoacoustic coupling regime.

Figure 4(a) compares the splitting ratio $p$ for different waveguides and different pump power. The blue dashed curve corresponds to a silica tapered fiber with a $1 \mu \mathrm{m}$ mode-field diameter. As can be seen, the splitting ratio $p$ never exceeds one. The green dotted line represents a similar tapered fiber made of $\mathrm{As}_{2} \mathrm{Se}_{3}$ chalcogenide glass. Thanks to the strong Brillouin gain, the Rabi splitting occurs for pump powers superior to a few watts. Finally, the blue dashed curve represents what we would expect from a suspended silicon nanowire that would experience the giant enhancement of SBS gain predicted in Refs. [18,19]. Note that whether backward
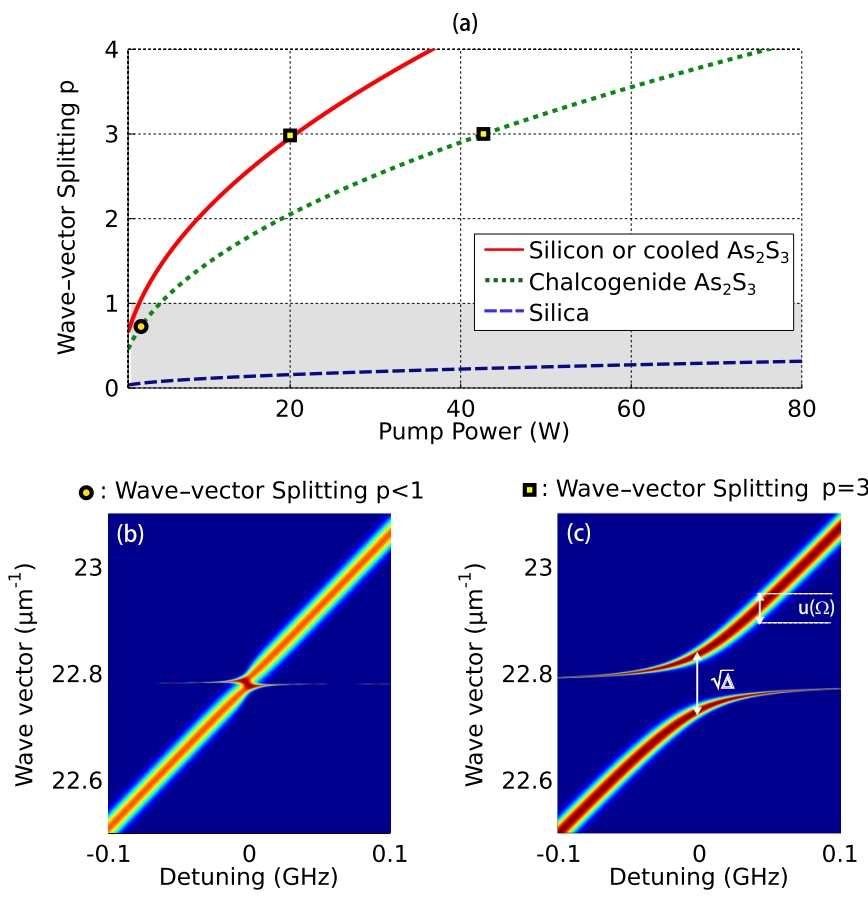

FIG. 4. (a) Wave-vector splitting ratio $p$ for silicon nanowire or cooled $\mathrm{As}_{2} \mathrm{Se}_{3}$ (solid line), room temperature $\mathrm{As}_{2} \mathrm{Se}_{3}$ (dotted line), and silica (dashed line) fiber taper vs the pump power. $p$ measures the gap between two split curves in number of linewidth. The gray area delimits the weak-coupling regime. All of the parameters used to make the calculation are listed in Appendix E. (b) Dispersion curves $K_{ \pm}(\Omega)$ for $2.5 \mathrm{~W}$ pump power and $1 \mathrm{~mW}$ Stokes power in a $1 \mu \mathrm{m}$ mode-field diameter tapered $\mathrm{As}_{2} \mathrm{Se}_{3}$ fiber. (d) Dispersion curve $K_{ \pm}(\Omega)$ for $20 \mathrm{~W}$ pump power and $1 \mathrm{~mW}$ Stokes power in a silicon nanowire as described in [19] or for $43 \mathrm{~W}$ pump power in the previous tapered $\mathrm{As}_{2} \mathrm{Se}_{3}$ fiber. Optical loss is $1 \mathrm{~dB} \mathrm{~m}^{-1}$.

Brillouin scattering can be observed in silicon nanowire is still under debate, but it does not question our theory since a similar curve can be readily obtained with the previous chalcogenide tapered fiber provided that the Brillouin linewidth is reduced to $8 \mathrm{MHz}$. Le Floch and Cambon have demonstrated that such significant reduction can be achieved by cooling [22]. Figure 4(a) also illustrates the weak- and strong-coupling regimes for $p=0.75$ (circle in the gray area) and $p=3$ (square). In the weak-coupling regime $p<1$ shown in Fig. 4(b), the nonlinear process is not strong enough and no splitting can be observed.

On the contrary, Fig. 4(c) clearly shows a strong gap $\sqrt{\Delta}$ between the two branches. Rabi splitting occurs when the gap exceeds a certain number of Brillouin linewidths defined by $|u(\Omega)|$.

This number of linewidths is indeed the splitting ratio $p$ that is plotted in Fig. 4(a) as a function of the pump power. One can see that for a silica-based taper, the Brillouin gain is not strong enough to induce any splitting even at high pump power, whereas the chalcogenide fiber taper enables splitting from a pump power just above $5 \mathrm{~W}$. This is simply due to the fact that Brillouin gain in $\mathrm{As}_{2} \mathrm{Se}_{3}$ fiber taper is about 200 times that of silica and a smaller linewidth (see Appendix E). The splitting ratio is further illustrated in Fig. 4(c) for a silicon nanowire or a cooled chalcogenide fiber taper. In this case, $p=3$, we find a gap between the two branches of 3 linewidths. 


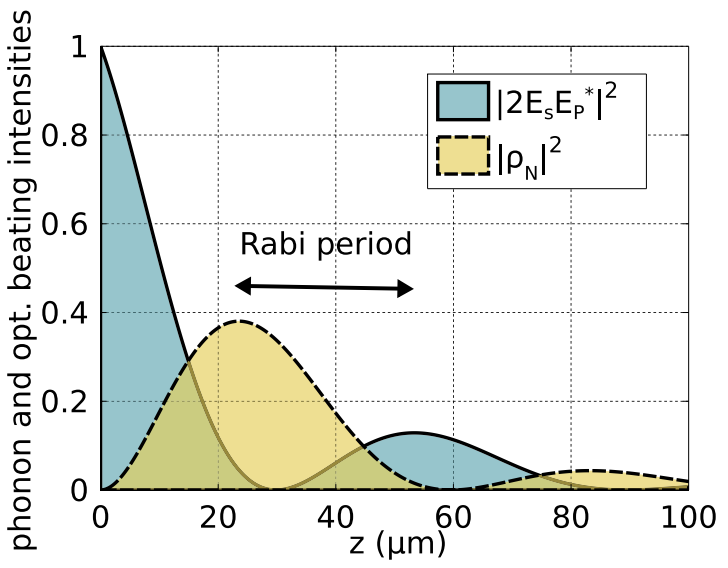

FIG. 5. Squared amplitude of the phonon and the optical beat vs the $z$ axis. We use $40 \mathrm{~W}$ pump power and $1 \mathrm{~mW}$ Stokes power in a $1 \mu \mathrm{m}$ mode-field diameter tapered $\mathrm{As}_{2} \mathrm{Se}_{3}$ fiber. The parameters are from Appendix E.

A further comparison between Figs. 3 and 4 shows that the optical beat branch vanishes in Fig. 4. This is due to the fact that the optical beat experiences negligible loss $\left(1 \mathrm{~dB} \mathrm{~m}^{-1}\right)$, and thus its branch is very thin and beyond image resolution. Unlike the optical beat, acoustic phonon experiences much larger loss, leading to a much wider branch than the optical one. Consequently, we can deduce that the resonant nature of the process is definitely related to the phonon lifetime.

Once we have seen how the splitting ratio $p$ evolves with the optical power, it is interesting to investigate the influence of the waveguide geometry. It is indeed inversely proportional to $\sqrt{A_{\text {eff }}}$, making it stronger for submicron waveguides. However, for such dimension, one has to pay attention to the radiation pressure due to strong electric-field variation at the waveguide boundaries. For relatively modest refractive index contrast, as in chalcogenide glasses $(n=2.4)$, radiation pressure can be considered negligible for a $1 \mu \mathrm{m}$ mode-field diameter (MFD). Radiation pressure has a noticeable impact in high-confinement waveguides such as silicon nanowires with few-hundred-nm width and high-index contrast $\left(n_{\mathrm{Si}}=3.4\right)$ and can be taken to our advantage $[19,23,24]$. These effects could be more explicitly taken into account using another set of coupled equations similar to Boyd's model including radiation pressure [24] or by the introduction of the Rakich et al. Brillouin parameter, as we did in Fig. 4(a) (see Appendix A for detailed explanation). In the next section, we will investigate the spatial oscillating behavior that results from the Rabi splitting.

\section{SPATIAL BEHAVIOR}

Following the computation of the eigenvalues depicted in Fig. 3, we can calculate the spatial behavior. At the degeneracy frequency, the eigenvectors are $\left(1, \frac{\sqrt{\Delta}}{k_{o p}}\right)$ and $\left(1,-\frac{\sqrt{\Delta}}{k_{o p}}\right)$. Normalizing $\tilde{\rho}$ to $\tilde{\rho}_{N}=\tilde{\rho} \frac{k_{o p}}{\sqrt{\Delta}}$, the new eigenvectors are $(1,1)$ and $(1,-1)$. The damped oscillations of the two eigensolutions are depicted in Fig. 5. This figure shows that the direct sum of both eigenvectors leads to the initial conditions where there is no phonon and maximum optical beat intensity. Then the normalized phonon intensity rises as the optical beat vanishes.

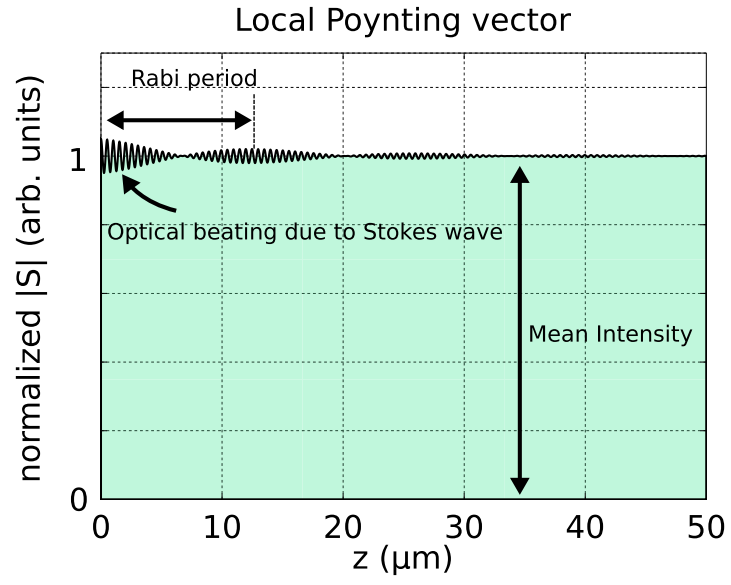

FIG. 6. Scheme of the local Poynting vector oscillation due to the strong coupling with a single acoustic wave. Note that the fringe's visibility reaches zero with full contrast, which may not always be the case, depending on the initial conditions. The optical beat amplitude is overemphasized for better visibility.

After a few microns, the process is reversed as the system oscillates with a Rabi period of a dozen microns. As illustrated by Fig. 3, the oscillation must be faster than the acoustic decay rate which is satisfied if the splitting ratio $p>1$.

Here, the "intensity" of the optical beat $\left|\widehat{\tilde{E}_{P} \tilde{H}_{S}^{*}}\right|^{2}$ is not related to the optical mean power. The optical beat is a local fluctuation of the optical intensity, in other words, a set of interference fringes. The oscillation of $\left|\widetilde{\tilde{E}_{P} \widetilde{H}_{S}^{*}}\right|^{2}$ is thus an oscillation of the fringe's visibility. As explained in Table I, we do not describe a process phase matched to provide optical gain or depletion. As a result, the two first terms of the Poynting vector in Eq. (9) that are related to the pump and Stokes power remain constant. It is also confirmed by the fact that in this regime, the RHS of Eqs. (26) and (27) is zero. This is shown schematically in Fig. 6 by the strong constant mean value of the local Poynting vector. On top of that, the oscillation of the third term of the Poynting vector $\mid{\widetilde{\tilde{E}_{P}} \widetilde{H}_{S}^{*}}^{2}$ in Eq. (9) translates in the oscillation of the optical beat amplitude and thus a periodic alteration of the visibility of the interference. The consequence is that the strong-coupling regime corresponds to the periodic loss of visibility of the optical beat. Note that for better visibility of Fig. 6, we have limited ourselves to one acoustic wave and overemphasized the optical beat amplitude fluctuation. In reality, the optical intensity is much more important compared to the local fluctuation and the fringes result from the superposition of many acoustic waves along $z$.

\section{CONCLUSION}

In conclusion, we have demonstrated from the theory of Brillouin scattering in optical waveguides that strong coupling can be achieved between the acoustic wave and the two optical waves, giving rise to anticrossing and Rabi-like splitting. This was demonstrated by deriving from the coupled equations of BS a joint system that combines both the phonon and the optical beat note resulting from the coherent superposition of the pump and Stokes waves. It has been shown that when the nonlinear coupling rate becomes comparable to 
the phonon decay rate, the joint system enters the strongcoupling regime and exhibits two split eigenvectors $K_{ \pm}(\Omega)$. We found numerically that this regime should be accessible in highly nonlinear subwavelength waveguides. Finally, this work contributes to the further understanding of strong photonphonon interactions and also proves that effects familiar to cavity optomechanics can also occur in Brillouin scattering, as recently suggested in Ref. [12].

\section{ACKNOWLEDGMENTS}

This work was supported by the OASIS Project No. ANR-14-CE36-0005-01, the support from the Région de Franche-Comté, and the LABEX ACTION Program No. ANR-11-LABX-0001-01.

\section{APPENDIX A: PHASE-MATCHING MAP FOR CHALCOGENIDE FIBER}

In Fig. 7, we report the same calculation as in Fig. 2, but for a chalcogenide fiber taper using parameters in Appendix E. Compared to silica, the lower acoustic velocity. allows a greater number of acoustic modes and a bulk frequency around $8 \mathrm{GHz}$.

\section{APPENDIX B: NORMALIZATION AND SUBMICRON TREATMENT}

The fields are normalized so that the modulus $\left|2 \tilde{A} \tilde{B}^{*}\right|$ in Eq. (12) is the optical power. Thus the effective distance $D_{\text {eff }}$ can be written as

$$
D_{\mathrm{eff}}=\frac{\iint\left|E_{S}\right|^{2} d x d y \sqrt{\iint|R|^{2} d x d y}}{\iint \mathcal{R}(x, y) E_{S} E_{P}^{*} d x d y}=\sqrt{\pi} \sigma,
$$

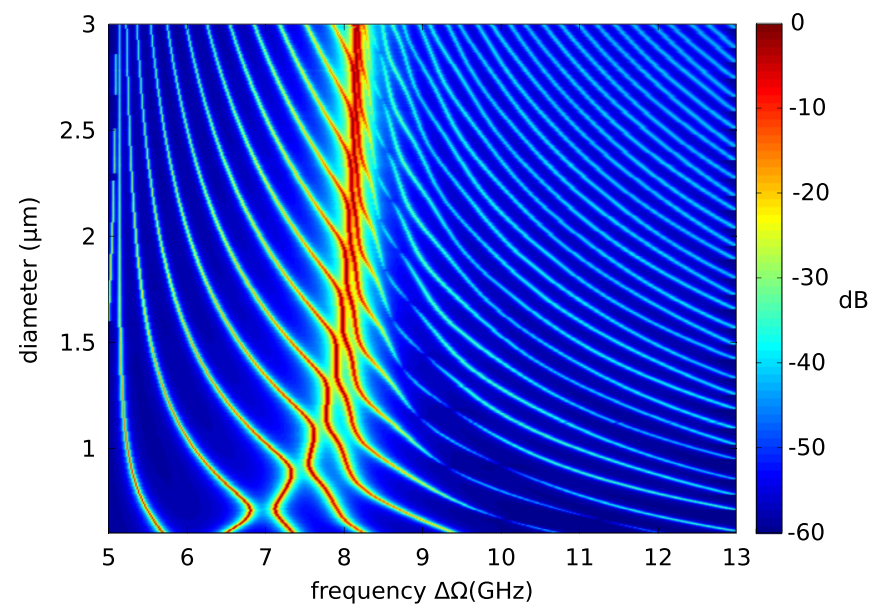

FIG. 7. Color plot of the Brillouin spectrum related to the coupling strength between longitudinal acoustic modes with the fundamental optical mode of a silica fiber taper as a function of the acoustic frequency (horizontal axis) and of the taper diameter (vertical axis). The color scale describes the normalized overlap between the acoustic and optical modes. The absolute effective area $A_{\text {eff }}$ is not taken into account; it would show an enhancement for small diameters. where $\sigma=\frac{\text { MFD }}{2}$ is the waist and MFD is the mode-field diameter (after Ref. [25]). We can also define the effective mode area as $A_{\text {eff }}=D_{\text {eff }}^{2}$. In this case, we assume that both pump and stokes lights are in the fundamental mode of the waveguide.

Note that this is a standard treatment of the transverse fields and that the specific case of submicron waveguides requires more complex calculations without changing the main results of this paper. In standard fibers, the main contribution of electrostriction comes from one component of the stress tensor. In submicron devices, all of the components contribute to this effect. Moreover, in high-index nanowires, the continuity of the normal component of the displacement vector induces strong discontinuities of the electric field. It leads to an enhancement of radiation pressure that must be taken into account. All of those aspects have been treated in Refs. [19,26] and do not fundamentally question our model. Equations (14)-(16) in [26] are very similar to the nonlinear coupled mode equation system that we have used. The main difference is that one has to sum the contributions of all the components of the electric field and all the components of the displacement vector. In our case, we consider optical and acoustic modes. It means that all the components $\left(E_{z}, E_{\theta}, E_{r}, H_{z}, H_{\theta}, H_{r}\right)$ of the optical mode are coherent and bound by the dispersion equation (1). Since the mode is an electromagnetic eigensolution of the waveguide, all those components are proportional to one amplitude related to the optical power carried by the optical mode. As a result, the different components of the electrostriction tensor in Ref. [26] can be simplified by factorizing the optical mode amplitude. Similarly, the same treatment can be applied to the acoustic mode. We can factorize the acoustic mode amplitude from all of the components of the displacement vector $\left(u_{k, l}\right)$. After factorization, we find a standard nonlinear coupled mode equation similar to the one in Boyd [20], where only the optical and acoustic mode amplitude appear. The only difference comes from a term that replaces $\frac{\gamma_{e}}{D_{\text {eff }}}$ that eventually results from the sum of all tensorial contributions. The same arguments apply to radiation pressure. For example, in Fig. 4, we use the Brillouin parameter calculated by Rakich et al. [19] and replace $\frac{g}{A_{\text {eff }}}$ in our model to take those considerations into account.

\section{APPENDIX C: GROUP VELOCITY OF THE OPTICAL INTERFERENCE PATTERN}

If we do not neglect the group velocity in Eq. (12), we get

$$
\begin{aligned}
& \frac{\partial \tilde{A}_{P} \tilde{B}_{S}^{*}}{\partial z}+\frac{\partial \beta}{\partial \omega}\left\{\frac{\partial \tilde{A}_{P}}{\partial t} \tilde{B}_{S}^{*}-\tilde{A}_{P} \frac{\partial \tilde{B}_{S}^{*}}{\partial t}\right\} \\
& =-\frac{i \omega_{P} \omega_{S} \gamma_{e} \tilde{\rho}}{2 n c \rho_{0} D_{\text {eff }}}\left(\frac{\tilde{A}_{P} \tilde{B}_{P}^{*}}{\omega_{P}}-\frac{\tilde{A}_{S} \tilde{B}_{S}^{*}}{\omega_{S}}\right) .
\end{aligned}
$$

Note that if the two pump and Stokes waves were propagating along the same direction in the fundamental mode, the lefthand side (LHS) would become $\frac{\partial \tilde{A}_{P} \tilde{B}_{S}^{*}}{\partial z}+\frac{\partial \beta}{\partial \omega} \frac{\partial \tilde{A}_{P} \tilde{B}_{S}^{*}}{\partial t}$, giving rise to a forward optical beat with its envelope traveling at the group velocity. In the counterpropagating case, it is much more difficult to define a group velocity for the optical interference pattern. However, we can show that the effective group velocity is somehow bounded and has a negligible effect on our main 
(d)
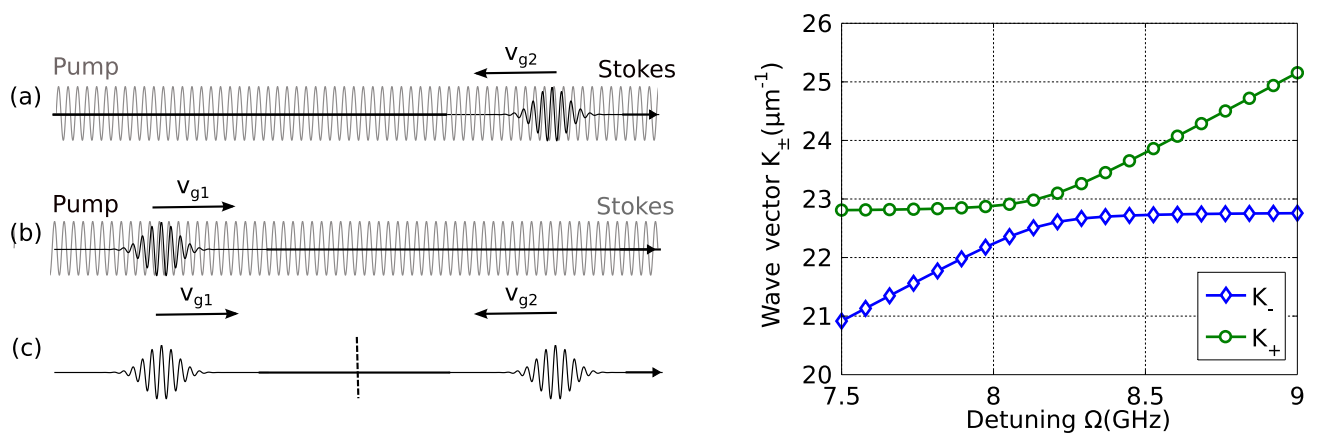

FIG. 8. (a) Pump in gray is a continuous wave and travels forward along the $z$ axis; Stokes pulse light travels backward. (b) Pump pulse in black travels forward along the $z$ axis; Stokes light in gray is continuous and travels backward. (c) Pump and Stokes are pulses and travel in opposite directions. The resulting wave packet is static. (d) Same computation as in Fig. 3, but with $\frac{1}{v_{g}}=-\frac{\partial \beta}{\partial \omega}$ in green (circles) and blue (diamonds) and with $\frac{1}{v_{g}}=+\frac{\partial \beta}{\partial \omega}$ in markers.

results. We can see in Fig. 8(a) three different scenarios. First, if the pump is a continuous wave, $\frac{\partial \tilde{A}_{P}}{\partial t}=0$, and the Stokes wave is an optical pulse, the intensity fluctuations due to the superposition of both waves are located at the same location as the Stokes pulse. The optical beat pattern thus follows the Stokes pulse and propagates at the same group velocity. Note that the interaction with the phonon is not likely to disturb this equilibrium if the pump is sufficiently strong compared to the Stokes wave, $\left|\tilde{A}_{P}\right| \gg\left|\tilde{A}_{S}\right|$. Figure 8(b) shows the opposite scenario where the Stokes is a continuous wave and the pump a pulse. In this case, the optical beat follows the pump pulse and its group velocity is therefore the pump group velocity. The striking consequence is that this group velocity is the opposite of the previous case, as pump and Stokes propagate in opposite direction. From the equation, we can address a third interesting example: if $\tilde{A}_{P}=\tilde{B}_{S}^{*}$, the group velocity of the optical beat is then null. This is depicted in Fig. 8(c), where both pump and Stokes waves are optical pulses and the barycenter of both pulses is static. This last case is, however, very unlikely to happen since an increase of $\tilde{B}_{S}^{*}$ over time due to the phonon is clearly related to a depletion of the pump $\tilde{A}_{P}$ with much impact on the group velocity.

As a result, if the group velocity of the optical interference pattern may be uncertain, it is certainly bounded by the relation $\frac{1}{v_{g}} \in\left[-\frac{\partial \beta}{\partial \omega},+\frac{\partial \beta}{\partial \omega}\right]$, where the limits correspond to the first two cases. More intriguingly, though these boundaries are wide apart, they do not affect our main results. Figure 8(d) shows a similar numerical simulation of the anticrossing as in Fig. 3, but with $\frac{1}{v_{g}}=-\frac{\partial \beta}{\partial \omega}$ in green (circles) and blue (diamonds) and $\frac{1}{v_{g}}=+\frac{\partial \beta}{\partial \omega}$ with markers. There is no noticeable difference because $\left|\frac{1}{v_{g}}\right| \simeq\left|\frac{n}{c}\right|$ is very small compared to $\left|\frac{1}{v_{a}}\right|$ and thus negligible, whatever the sign is. The second point we want to stress is that in most Brillouin experiments, the group velocity is always positive since the pump power is much greater than the Stokes power and does not suffer from depletion. Such assumption leads to $\left|\frac{\partial \tilde{A}_{P}}{\partial t} \tilde{B}_{S}^{*}\right| \ll\left|\tilde{A}_{P} \frac{\partial \tilde{B}_{S}^{*}}{\partial t}\right|$, and we can thus write

$$
\frac{\partial \tilde{A}_{P} \tilde{B}_{S}^{*}}{\partial t}=\frac{\partial \tilde{A}_{P}}{\partial t} \tilde{B}_{S}^{*}+\tilde{A}_{P} \frac{\partial \tilde{B}_{S}^{*}}{\partial t} \simeq+\tilde{A}_{P} \frac{\partial \tilde{B}_{S}^{*}}{\partial t},
$$

which is a positive group velocity.

\section{APPENDIX D: BRILLOUIN GAIN}

The Brillouin gain was defined using the method described in [27]. From the above Eqs. (6) and (11), we find

$$
\begin{aligned}
\frac{\partial \tilde{A}_{P} \tilde{B}_{P}^{*}}{\partial z}+\frac{\partial \beta}{\partial \omega} \frac{\partial \tilde{A}_{P} \tilde{B}_{P}^{*}}{\partial t} & =\kappa\left(\tilde{\rho} \tilde{A}_{S} \tilde{B}_{P}^{*}+\tilde{\rho}^{*} \tilde{A}_{P} \tilde{B}_{S}^{*}\right), \\
-\frac{\partial \tilde{A}_{S} \tilde{B}_{S}^{*}}{\partial z}+\frac{\partial \beta}{\partial \omega} \frac{\partial \tilde{A}_{S} \tilde{B}_{S}^{*}}{\partial t} & =\kappa\left(\tilde{\rho}^{*} \tilde{A}_{P} \tilde{B}_{S}^{*}+\tilde{\rho} \tilde{A}_{S} \tilde{B}_{P}^{*}\right) .
\end{aligned}
$$

These equations first show that the optical powers propagate at the same group velocity. Moreover, if we neglect the nonlinear coupling term and then subtract both equations, we can use the Poynting theorem for plane waves, $\operatorname{div} \vec{S}=0$, to remove the spatial derivative and get the conservation law $\frac{\partial \tilde{A}_{S} \tilde{B}_{S}^{*}}{\partial t}-$ $\frac{\partial \tilde{A}_{P} \tilde{B}_{P}^{*}}{\partial t}=0$, or $\frac{\partial P_{S}}{\partial t}+\frac{\partial P_{P}}{\partial t}=0$. If the nonlinear coupling term is kept, we must integrate from one fiber end to the other. In the steady-state condition, we get $P_{S}(L)+P_{P}(0)+P_{\rho}(0)=$ $P_{S}(0)+P_{P}(L)+P_{\rho}(L)$ that ensures the energy conservation.

Using the phonon equation (14) in the steady-state regime, in order to substitute $\tilde{\rho}$, we find

$$
\tilde{\rho}(z, t)=-\frac{1}{\Omega_{B}^{2}-\Omega^{2}-i \Omega \Gamma_{B}} \frac{\gamma_{e} \beta_{a}^{2}}{n c} \frac{1}{D_{\mathrm{eff}}} \tilde{A}_{P} \tilde{B}_{S}^{*},
$$

and assuming $\omega_{P} \simeq \omega_{S}$, we thus obtain the usual power equation evolution of SBS, as in Ref. [27],

$$
\begin{aligned}
\frac{\partial P_{P}}{\partial z} & =-\frac{g}{A_{\mathrm{eff}}} P_{P} P_{S}, \\
\frac{\partial P_{S}}{\partial z} & =\frac{g}{A_{\mathrm{eff}}} P_{P} P_{S},
\end{aligned}
$$

where $A_{\text {eff }}=D_{\text {eff }}^{2}, \kappa_{1}=\frac{\omega_{s} \gamma_{e}}{2 n_{S} c \rho_{0}}$, and $\kappa_{2}=\frac{\gamma_{e} \beta_{a}^{2}}{2 n c} \frac{1}{\Omega}=\frac{\gamma_{e} \omega_{S}}{c^{2} v_{a}}$, with

$$
g(\Omega)=g_{0} \frac{\left(\Gamma_{B} / 2\right)^{2}}{\left(\Omega_{B}-\Omega\right)^{2}+\left(\Gamma_{B} / 2\right)^{2}}
$$

where $g_{0}=4 \kappa_{1} \kappa_{2} \frac{1}{\Gamma_{B}}=2 \frac{\omega_{S} \omega_{P} \gamma_{e}^{2}}{n_{S} c^{3} v_{a} \rho_{0} \Gamma_{B}}$ is the Brillouin gain [27]. 
TABLE II. Table of optical and acoustical parameters of silicabased and chalcogenide-based glass materials and silicon.

\begin{tabular}{lccc}
\hline \hline & Silica $^{\mathrm{a}}$ & $\mathrm{As}_{2} \mathrm{Se}_{3}{ }^{\mathrm{b}}$ & Silicon $^{\mathrm{c}}$ \\
\hline Refractive index at $1.5 \mu \mathrm{m}$ & 1.45 & 2.8 & 3.5 \\
Brillouin frequency shift $(\mathrm{GHz})$ & 11 & 8 & 39 \\
Brillouin linewidth $(\mathrm{MHz})$ & 30 & 13 & 40 \\
Acoustic velocity $\left(\mathrm{m} \mathrm{s}^{-1}\right)$ & 5600 & 2250 & 8433 \\
Brillouin gain $\left(\mathrm{m} \mathrm{W}^{-1}\right)$ & $3.3 \times 10^{-11}$ & $600 \times 10^{-11}$ & $\mathrm{~d}$ \\
Mean mass density $\left(\mathrm{Kg} \mathrm{m}^{-3}\right)$ & 2200 & 4640 & 2328 \\
\hline \hline
\end{tabular}

${ }^{\mathrm{a}}$ See [27].

${ }^{\mathrm{b}}$ See $[29,30]$.

${ }^{\mathrm{c}}$ See $[16,19]$.

${ }^{\mathrm{d}}$ Since silicon nanowires are very sensitive to radiation pressure, it is not convenient to separate the geometry of the waveguide from the Brillouin gain. Rakich et al. predicts Brillouin parameters up to $10^{4} \mathrm{~m}^{-1} \mathrm{~W}^{-1}$ for $300 \times 280 \mathrm{~nm}$ effective area nanowires for resonances around $13 \mathrm{GHz}$ [19].

\section{APPENDIX E: MATERIAL PARAMETERS}

In our simulations, we used and compared silica-based fiber tapers and chalcogenide $\mathrm{As}_{2} \mathrm{Se}_{3}$ glass-based fiber tapers, as they are readily available and manufactured [28]. Moreover, recent experiments have shown the great potential of these glass materials for SBS applications [13,14]. In addition, much attention has recently been paid to silicon photonics with the prediction of giant enhancement of the Brillouin gain through the radiation pressure [19]. Table II summarizes the optical and acoustical parameters of these three materials. Note that the $\mathrm{As}_{2} \mathrm{Se}_{3}$ Brillouin linewidth can be as low as $13 \mathrm{MHz}$ when no substrate or coating is used $[13,29,30]$.

\section{APPENDIX F: ANTI-STOKES SCATTERING}

One of the main hypotheses for the strong-coupling Brillouin regime was to consider both the anti-Stokes and

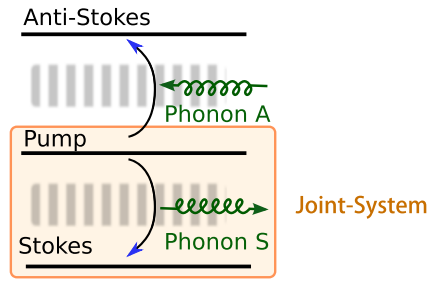

FIG. 9. Scheme of the anti-Stokes scattering process above the joint pump-Stokes system.

second-order Stokes scattering as negligible. This assumption is valid in the weak pump-to-Stokes conversion regime. However, adding the anti-Stokes wave as a higher-energy level in the system equation does not significantly change our results. As shown in Fig. 9, anti-Stokes scattering involves an oncoming acoustic phonon $A$ that must not be mistaken for the forwardpropagating phonon $B$, involved in the joint pump-Stokes system [20]. The scheme illustrates that antiStokes scattering can then be considered as an additional loss for the pump level. As a result, the model could be considered as a three-level system [5]. The pump depletion due to this phenomenon would then be associated with a decay rate related to the room-temperature phonon population. However, in optical fibers, anti-Stokes scattering is much weaker than Stokes scattering due to the low-phonon population and the fast phonon decay rate. Therefore, it is usually neglected, particularly in the stimulated regime [31]. In the strongcoupling regime, the coupling must be stronger than the phonon decay that is around a few tens of microns. Over such a short distance, the pump decay rate associated with anti-Stokes scattering is thus negligible. Note that second-order Stokes scattering can be neglected for the same reasons. For example, experiments were reported with $3.5 \mathrm{~ns}$ pulses with peak power $40 \mathrm{~W}$ in $10-\mathrm{cm}$-long and 6- $\mu \mathrm{m}$-diameter $\mathrm{As}_{2} \mathrm{Se}_{3}$ fiber, where the second-order Stokes peak is $20 \mathrm{~dB}$ smaller than the first order [32].
[1] M. Aspelmeyer, T. J. Kippenberg, and F. Marquardt, Cavity Optomechanics (Springer, Berlin, 2014).

[2] M. Aspelmeyer, T. J. Kippenberg, and F. Marquardt, Rev. Mod. Phys. 86, 1391 (2014).

[3] G. Bahl, M. Tomes, F. Marquardt, and T. Carmon, Nat. Phys. 8, 203 (2012).

[4] J. H. Kim, M. C. Kuzyk, K. Han, H. Wang, and G. Bahl, Nat. Phys. 11, 275 (2015).

[5] S. Haroche and J-M. Raimond, Exploring the Quantum: Atoms, Cavities, and Photons (Oxford University Press, Oxford, 2006), p. 204.

[6] H. Deng, H. Haug, and Y. Yamamoto, Rev. Mod. Phys. 82, 1489 (2010).

[7] S. Gröblacher, K. Hammerer, M. R. Vanner, and M. Aspelmeyer, Nature (London) 460, 724 (2009).

[8] E. Verhagen, S. Deléglise, S. Weis, A. Schliesser, and T. J. Kippenberg, Nature (London) 482, 63 (2012).

[9] A. V. Zayats, I. I. Smolyaninov, and A. A. Maradudin, Phys. Rep. 408, 131 (2005).
[10] J. Le Gall, M. Olivier, and J.-J. Greffet, Phys. Rev. B 55, 10105 (1997).

[11] A. Wallraff, D. I. Schuster, A. Blais, L. Frunzio, R.-S. Huang, J. Majer, S. Kumar, S. M. Girvin, and R. J. Schoelkopf, Nature (London) 431, 162 (2004).

[12] R. Van Laer, R. Baets, and D. Van Thourhout, Phys. Rev. A 93, 053828 (2016).

[13] J.-C. Beugnot, R. Ahmad, M. Rochette, V. Laude, H. Maillotte, and T. Sylvestre, Opt. Lett. 39, 482 (2014).

[14] J.-C. Beugnot, S. Lebrun, G. Pauliat, H. Maillotte, V. Laude, and T. Sylvestre, Nat. Commun. 5, 5242 (2014).

[15] D. Royer and E. Dieulesaint, Elastic Waves in Solids I - Free and Guided Propagation (Springer, Berlin, 2000).

[16] R. Van Laer, B. Kuyken, D. Van Thourhout, and R. Baets, Nat. Photon. 9, 199 (2015).

[17] A. W. Snyder and J. D. Love, Optical Waveguide Theory (Springer, Boston, MA, 1984).

[18] P. T. Rakich, Z. Wang, and P. Davids, Opt. Lett. 36, 217 (2011). 
[19] P. T. Rakich, C. Reinke, R. Camacho, P. Davids, and Z. Wang, Phys. Rev. X 2, 011008 (2012).

[20] R. W. Boyd, Nonlinear Optics, 3rd ed. (Academic, Burlington, MA, 2008).

[21] Y. Yamamoto, F. Tassone, and H. Cao, Semiconductor Cavity Quantum Electrodynamics (Springer, Berlin, 2000).

[22] S. Le Floch and P. Cambon, Opt. Commun. 219, 395 (2003).

[23] H. Shin, W. Qiu, R. Jarecki, J. A. Cox, R. H. Olsson III, A. Starbuck, Z. Wang, and P. T. Rakich, Nat. Commun. 4, 1944 (2013).

[24] C. Wolff, M. J. Steel, B. J. Eggleton, and C. G. Poulton, Phys. Rev. A 92, 013836 (2015).

[25] K. Miyagi, Y. Namihira, S. M. A. Razzak, S. F. Kaijage, and F. Begum, Opt. Rev. 17, 388 (2010).
[26] Y. Pennec, V. Laude, N. Papanikolaou, B. Djafari-Rouhani, M. Oudich, J. S. El, J. C. Beugnot, J. M. Escalante, and A. Martnez, Nanophotonics 3, 413 (2014).

[27] G. Agrawal, Nonlinear Fiber Optics, 4th ed. (Academic, Amsterdam, 2006).

[28] C. Baker and M. Rochette, IEEE Photon. J. 4, 960 (2012).

[29] K. S. Abedin, Opt. Express 13, 10266 (2005).

[30] R. Pant, C. G. Poulton, D.-Y. Choi, H. Mcfarlane, S. Hile, E. Li, L. Thevenaz, B. Luther-Davies, S. J. Madden, and B. J. Eggleton, Opt. Express 19, 8285 (2011).

[31] A. Kobyakov, M. Sauer, and D. Chowdhury, Adv. Opt. Photon. 2, 1 (2010).

[32] T. F. S. Büttner, I. V. Kabakova, D. D. Hudson, R. Pant, E. Li, and B. J. Eggleton, Opt. Express 20, 26434 (2012). 